\title{
In vitro sensitivity to platinum-derived drugs is associated with expression of thymidylate synthase and dihydropyrimidine dehydrogenase in human lung cancer
}

\author{
MASAYA TAKIZAWA ${ }^{1}$, KAZUYUKI KAWAKAMI ${ }^{1}$, TOHRU OBATA ${ }^{2}$, ISAO MATSUMOTO ${ }^{1}$, \\ YASUHIKO OHTA $^{1}$, MAKOTO ODA $^{1}$, TAKUMA SASAKI ${ }^{2}$ and GO WATANABE ${ }^{1}$ \\ ${ }^{1}$ Department of General and Cardiothoracic Surgery, Kanazawa University Graduate School of Medicine; ${ }^{1}$ Department of \\ Experimental Therapeutics, Kanazawa University Cancer Research Institute, Takaramachi 13-1, Kanazawa 920-8641, Japan
}

Received November 21, 2005; Accepted January 23, 2006

\begin{abstract}
Thymidylate synthase (TS) and dihydropyrimidine dehydrogenase (DPD) are critical enzymes in nucleic acid metabolism. Proliferating cell nuclear antigen (PCNA) is a specific protein that is correlated with proliferative activity of cells. The TS gene has a variable number of tandem repeats (VNTR) in its 5'-untranslated region and a single nucleotide polymorphism (SNP) in the VNTR area. We examined the association of in vitro sensitivity to anticancer drugs with TS polymorphism, TS, DPD, and PCNA mRNA expression using human lung cancer tissues. Seventy-eight surgically resected lung cancer tissues were tested for in vitro sensitivity to 5fluorouracil, cisplatin (CDDP), carboplatin (CBDCA), irinotecan, docetaxel, and gemcitabine by histoculture and MTT assay. The TS polymorphisms were analyzed by PCR and PCR-RFLP. TS, DPD, and PCNA mRNA expression levels were quantified by real-time RT-PCR and normalized relative to $\beta$-actin mRNA expression. The inhibition rates (IRs) of CDDP and CBDCA were significantly correlated with TS/ PCNA, the ratio of TS/actin and PCNA/actin, and DPD/PCNA, the ratio of DPD/actin and PCNA/actin. This correlation was further explored by subgroup analyses according to TS VNTR or TS functional type, in which $2 \mathrm{R} / 3 \mathrm{G}, 3 \mathrm{C} / 3 \mathrm{G}$, or $3 \mathrm{G} / 3 \mathrm{G}$ were classified into $\mathrm{H}$-type group and $2 \mathrm{R} / 2 \mathrm{R}, 2 \mathrm{R} / 3 \mathrm{C}$, or $3 \mathrm{C} / 3 \mathrm{C}$ into L-type group. The associations of TS/PCNA and DPD/PCNA with the IRs of CDDP, CBDCA remained significant in the $3 \mathrm{R} / 3 \mathrm{R}$ group and H-type group. These results suggest that in vitro sensitivity to platinum-derived drugs, CDDP and CBDCA, is associated with PCNA-normalized mRNA expression of TS and DPD in human lung cancer
\end{abstract}

Correspondence to: Dr Kazuyuki Kawakami, Department of General and Cardiothoracic Surgery, Kanazawa University Graduate School of Medicine, Takaramachi 13-1, Kanazawa 920-8641, Japan

E-mail: kawakami@med.kanazawa-u.ac.jp

Key words: thymidylate synthase, dihydropyrimidine dehydrogenase, proliferating cell nuclear antigen, chemosensitivity, lung cancer tissues, as affected by the TS polymorphism. The clinical significance of these pharmacogenomic markers for chemotherapy regimens with platinum-derived drugs should be investigated further for personalized treatment of lung cancer.

\section{Introduction}

Lung cancer is the leading cause of death from cancer worldwide (1). Despite radical surgery therapy, the cumulative 5-year survival rate of patients with lung cancer is $57-79 \%$ even for stage I disease $(2,3)$. Therefore, effective adjuvant chemotherapy is necessary in the treatment of lung cancer. Recent clinical trials suggested that post-operative adjuvant chemotherapy confers a survival benefit $(4,5)$, and this is becoming the standard strategy for the treatment of lung cancer. One of the problems associated with adjuvant chemotherapy is that long-term survival is the only marker of the effectiveness, which means that all patients undergo therapy with the full duration of the protocol as long as it is tolerable. Thus, some of the patients receive onerous treatment in vain, which has an adverse effect on the quality of their life. To avoid ineffective treatment, it is important to predict the tumor's sensitivity to anticancer drugs.

Thymidylate synthase (TS) catalyzes the reductive methylation of dUMP by 5,10-methylenetetrahydrofolate to form dTMP and dihydrofolate. TS is an important target for cancer chemotherapy because of its central, rate-limiting role in the de novo synthesis of dTTP (6). There have been a number of reports that TS expression is a predictive factor of the outcome of 5-fluorouracil (5-FU)-based chemotherapy $(7,8)$. The TS gene is known to have variable number of tandem repeats (VNTR) satellite sequences in its 5'-untranslated region (5'-UTR) (9), and this polymorphism is associated with TS protein expression $(10,11)$. Several clinical studies have indicated the association between TS VNTR genotype and the effectiveness of 5-FU-based chemotherapy $(12,13)$. More recently, a single nucleotide polymorphism (SNP) was identified in the VNTR area, and this was suggested to be a potential predictive factor for the effectiveness of chemotherapy (14). In addition to TS, dihydropyrimidine dehydrogenase (DPD) expression in cancer tissue has been analyzed extensively as a predictive marker of the effectiveness of 5-FU- 
based chemotherapy. DPD is the initial and rate-limiting enzyme in the chain of reactions in the catabolism of 5-FU. Accordingly, it has been suggested that high DPD activity is linked with a decrease in level of FdUMP, the active metabolite of 5-FU, resulting in a reduced effectiveness of the drug. Indeed, it has been reported that the DPD activity in tumor tissue is associated with sensitivity to 5-FU (8). These pharmacogenomic factors, TS expression, TS polymorphism, and DPD expression, have been analyzed mainly in gastrointestinal tumors and have attracted less attention in lung cancer because 5-FU was considered ineffective in this disease. However, recent clinical studies of UFT (tegafur and uracil) demonstrated that fluoropyrimidine is effective in lung cancer in an adjuvant setting (15-17), attracting more interest in TS and DPD as predictive factors.

Proliferating cell nuclear antigen (PCNA) is the specific protein that is correlated with the proliferative activity of cells. Several studies have suggested that PCNA expression may be an indicator of malignant potential in tumors because of its associations with the depth of invasion, organ metastasis, tumor differentiation, and tumor stage $(18,19)$. Therefore, PCNA expression is an independent prognostic factor in cancer patients and the combination of TS, DPD, and PCNA expression may be useful predictive factors in cancer chemotherapy.

Cisplatin, a key drug in the chemotherapy of lung cancer, exerts its anticancer activity through Pt-DNA adduct formation that inhibits DNA synthesis (20). High repair activity of the Pt-DNA adduct is a major mechanism of drug resistance to cisplatin (21). TS and DPD are key enzymes in nucleotide metabolism, which is essential for DNA repair. This reaction is critical as it maintains the essential metabolic requirements for cell proliferation and growth. Together, TS, DPD, and PCNA may be predictive factors of the efficacy of cisplatin as well as fluoropyrimidine. Furthermore, many other anticancer drugs target DNA synthesis, suggesting that TS, DPD, and PCNA may also be useful predictors of the efficacy of multiple anticancer agents used in lung cancer therapy.

In the present study, we studied TS polymorphism, TS, DPD, and PCNA mRNA expression, and in vitro sensitivity to various anticancer drugs using surgically resected non-small cell lung cancer specimens. The pharmacogenomic factors were evaluated by comparison with the results of in vitro sensitivity testing with 5-FU, cisplatin, carboplatin, irinotecan, docetaxel, and gemcitabine.

\section{Materials and methods}

Samples. A total of 78 cancer tissues were obtained surgically from 78 patients with primary non-small cell lung cancer (NSCLC), all of whom had given their written informed consent. All patients were Japanese and were comprised of 56 men and 22 women, ranging in age from 44 to 86 years, with a mean age of 66.0 years. Parts of the surgically obtained tissues were used for nucleic acid isolation and in vitro histoculture chemosensitivity testing with the 3-(4,5-dimethylthiazol2-yl)-2,5-diphenyl-2H tetrazolium bromide (MTT) assay. The remaining part of each sample was fixed with formalin and used for further histological examination to confirm the diagnosis postoperatively. All of the histological examinations were performed after staining with hematoxylin and eosin (H\&E). Ethical approval for the project was obtained from the Kanazawa University School of Medicine Ethics Committee.

Histoculture with anticancer drugs and the MTT assay. RPMI1640 medium containing $10 \%$ fetal bovine serum, $100 \mathrm{U} / \mathrm{ml}$ of penicillin, and $100 \mu \mathrm{g} / \mathrm{ml}$ of streptomycin was prepared as complete medium. Samples of about $1 \mathrm{~g}$ of the tumor tissues were washed in complete medium, followed by cutting into pieces of 1-2 $\mathrm{mm}$ in diameter as soon as possible. The pieces of the tumor tissue were then placed on collagen sponge gel manufactured from pig skin (Spongostan ${ }^{\circledR}$; Health Design Industries, Rochester, NY) and cultured in complete medium. Six anticancer agents, 5-fluorouracil (5-FU), cisplatin (CDDP), carboplatin (CBDCA), irinotecan (SN-38), docetaxel (DOC), and gemcitabine (GEM), were added to the culture medium. The concentrations of the anticancer agents were ten times the clinically achievable peak plasma concentrations (PPCs) in man calculated based on $\mathrm{LD}_{50}$ values in mice, which were defined as (22): $\log (\mathrm{PPC})=-0.788+\left[0.755 \mathrm{x} \log \left(\mathrm{LD}_{50}\right)\right]$.

After histoculture for 7 days, a modification of the MTT assay described by Mosmann (23) was performed. Briefly, aliquots of $40 \mu 1$ of MTT solution prepared by dissolving solid MTT $(2.0 \mathrm{mg} / \mathrm{ml})$ and $0.25 \%$ collagenase in phosphatebuffered saline (PBS) were added to each culture well and incubated at $37^{\circ} \mathrm{C}$ for $4 \mathrm{~h}$. The MTT-formazan products were dissolved in $0.3 \mathrm{ml}$ of DMSO. Then, the solutions were transferred to 96-well microplates and the absorbance was read at $540 \mathrm{~nm}$. The mean absorbance from triplicate assays was used to calculate the inhibition rate (IR) as follows $(24,25)$ :

$$
\begin{aligned}
& \text { Absorbance of the treated tumor / } \\
& \operatorname{IR}(\%)=\left[1-\frac{\text { weight of the treated tumor }(\mathrm{g})}{\text { Absorbance of the control tumor } /}\right] \quad \text { x100 } \\
& \text { weight of the control tumor }(\mathrm{g})
\end{aligned}
$$

In cases where the result calculated using this formula was less than $0 \%$, the IR was considered to be $0 \%$.

Nucleic acid isolation. Specimens of about $1 \mathrm{~g}$ of the tissues were stored immediately at $-80^{\circ} \mathrm{C}$ until DNA and RNA isolation. Genomic DNA was isolated using a QIAamp DNA Mini Kit ${ }^{\circledR}$ (Qiagen, Hilden, Germany) according to the protocol provided by the manufacturer's. Total RNA was isolated by the single-step guanidinium isothiocyanate method (26).

Analysis of TS genotypes. For analysis of the TS VNTR and G/C SNP in the 5'-UTR, PCR and PCR-RFLP methods were used as described previously $(10,14)$. Briefly, a fragment containing the repeats was amplified using the primers TS 25: 5'-AGGCGCGCGGAAGGGGTCCT-3' and TS 18: 5'TCCGAGCCGGCCACAGGCAT-3'. Aliquots of amplified fragments were separated on $3 \%$ agarose gels and the TS VNTR genotype was determined. Samples showing the $2 R / 3 R$ or $3 R / 3 R$ genotypes were further analyzed for the $G / C$ SNP by PCR-RFLP. HaeIII digestion of the $3 \mathrm{R}$ fragment produced bands of $66,37,28$, and $10 \mathrm{bp}$ for the $3 \mathrm{G}$ allele, and 94,37 and $10 \mathrm{bp}$ for the $3 \mathrm{C}$ allele after separation on $3 \%$ agarose gels. Accordingly, TS genotype was classified as 
Table I. Genotype frequency of TS polymorphism in NSCLC.

\begin{tabular}{|c|c|c|c|c|c|c|c|c|}
\hline & \multirow[t]{2}{*}{$2 \mathrm{R} / 2 \mathrm{R}$} & \multicolumn{2}{|c|}{$2 \mathrm{R} / 3 \mathrm{R}$} & \multicolumn{3}{|c|}{$3 R / 3 R$} & \multirow[t]{2}{*}{$3 R / 5 R$} & \multirow[t]{2}{*}{ Total } \\
\hline & & $2 \mathrm{R} / 3 \mathrm{G}$ & $2 \mathrm{R} / 3 \mathrm{C}$ & $3 \mathrm{G} / 3 \mathrm{G}$ & $3 \mathrm{G} / 3 \mathrm{C}$ & $3 \mathrm{C} / 3 \mathrm{C}$ & & \\
\hline Total & 2 & 13 & 15 & 14 & 21 & 12 & 1 & 78 \\
\hline \multicolumn{9}{|l|}{ Gender } \\
\hline Male & 1 & 11 & 10 & 12 & 15 & 7 & & 56 \\
\hline Female & 1 & 2 & 5 & 2 & 6 & 5 & 1 & 22 \\
\hline \multicolumn{9}{|l|}{ Histological type } \\
\hline Adeno & 1 & 11 & 9 & 8 & 14 & 8 & 1 & 52 \\
\hline Squamous cell & 1 & 2 & 5 & 6 & 15 & 3 & & 22 \\
\hline Others $^{\mathrm{a}}$ & & & 1 & & 2 & 1 & & 4 \\
\hline
\end{tabular}

a'Others contain adenosquamous, large cell, and mucoepidermoid carcinoma.

$2 \mathrm{R} / 2 \mathrm{R}, 2 \mathrm{R} / 3 \mathrm{G}, 2 \mathrm{R} / 3 \mathrm{C}, 3 \mathrm{G} / 3 \mathrm{G}, 3 \mathrm{G} / 3 \mathrm{C}$, or $3 \mathrm{C} / 3 \mathrm{C}$ based on the genotype of the VNTR and the SNP. Analysis was performed at least twice to confirm the genotype.

Quantification of TS, DPD, and PCNA mRNA. Quantification of the mRNA levels was carried out using a real-time fluorescence detection method as described previously (27). The quantities of TS, DPD, and PCNA mRNA were expressed as ratios relative to that of $\beta$-actin mRNA. The primer and probe sequences for TS and $B$-actin were listed previously (11). Those for DPD and PCNA were as follows: i) DPD: forward primer, GCAGACTC GAGACTGTAGGCACT; reverse primer, ACTCTCGATGTC CGCCGA; probe, FAM-CCATGGCCCCTGTGCTCAGTA AGG-TAMRA; ii) PCNA: forward primer, GTGCAAAAGAC GGAGTGAAATTT; reverse primer, ATCGACATTACTTG TCTGTGACAATTTA; probe, FAM-TGTTTCCATTTCCAA GTTCTCCACTTGCAG-TAMRA.

Statistical analysis. Groups were compared by the $\chi^{2}$ test and the Mann-Whitney U test. The correlations between TS, DPD, and PCNA mRNA expression and in vitro chemosensitivity were analyzed by Pearson's correlation coefficient test. $\mathrm{p}<0.05$ was considered to indicate significance.

\section{Results}

Frequencies of TS VNTR and G/C SNP in NSCLC. The genotype frequencies of TS polymorphisms are summarized in Table I. TS genotypes including repeat sequences longer than 3 were excluded from further analysis in this study because of their infrequency. There were no significant associations between the clinicopathological features, including patient's gender, age, and histological type, and genotype frequency of TS polymorphism.

TS, DPD, and PCNA mRNA expression in NSCLC. We quantified TS, DPD, and PCNA mRNA levels in 77 available samples from the 78 lung cancer tissues using a real-time fluorescence detection method. The histological type of the tumor was associated with DPD and PCNA mRNA expression.
Table II. TS, DPD, and PCNA mRNA expression in NSCLC.

No. TS/actin DPD/actin PCNA/actin

Total

$77 \quad 0.184 \pm 0.210 \quad 2.807 \pm 3.227 \quad 0.704 \pm 1.114$

Gender

Male

Female

$\begin{array}{llll}55 & 0.186 \pm 0.199 & 2.371 \pm 2.202 & 0.743 \pm 1.283\end{array}$

$22 \quad 0.178 \pm 0.240 \quad 3.898 \pm 4.846 \quad 0.606 \pm 0.491$ $\mathrm{p}=0.423 \quad \mathrm{p}=0.143 \quad \mathrm{p}=0.761$

Histological

type

$\begin{array}{lllll}\text { Adeno } & 51 & 0.141 \pm 0.099 & 3.502 \pm 3.658 & 0.443 \pm 0.347\end{array}$

$\begin{array}{llllll}\text { Squamous cell } 22 & 0.300 \pm 0.338 & 1.546 \pm 1.484 & 1.389 \pm 1.870\end{array}$ $\mathrm{p}=0.097 \quad \mathrm{p}=0.001 \quad \mathrm{p}<0.001$

Adenocarcinoma tissues showed higher levels of DPD/actin than squamous cell carcinoma tissues, and lower levels of PCNA/actin (Table II). There were no significant associations of the other clinicopathological features with TS, DPD, or PCNA mRNA expression. There was no relationship between TS, DPD, and PCNA mRNA expression and the malignant potential in tumors, including the size of the tumor, tumor differentiation, pleural invasion, vascular invasion, lymph nodes metastasis, and tumor stage (data not shown). There were no relationships between TS genotype and TS, DPD, or PCNA mRNA expression (data not shown).

Association of in vitro sensitivity to anticancer drugs with TS, $D P D$, and PCNA mRNA expression. The inhibition rates (IRs) of anticancer drugs described in Materials and methods were obtained in 68 samples for CDDP, CBDCA, 5-FU, and SN-38, 65 samples for DOC, and 66 samples for GEM. There were no significant associations of the IRs of any anticancer drugs with clinicopathological features, TS polymorphism, or the mRNA expression of TS, DPD, or PCNA. Next, we calculated the ratio of TS/actin and PCNA/actin, expressed as TS/PCNA. 


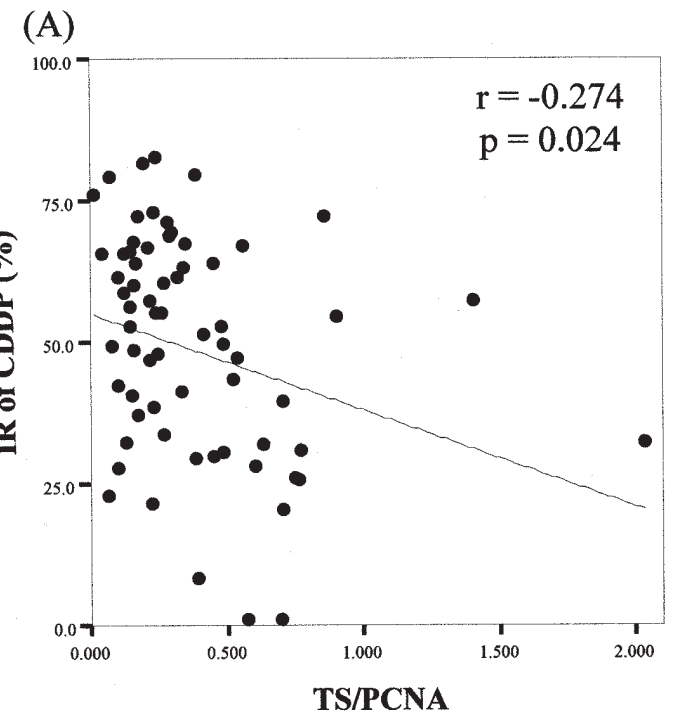

(B)

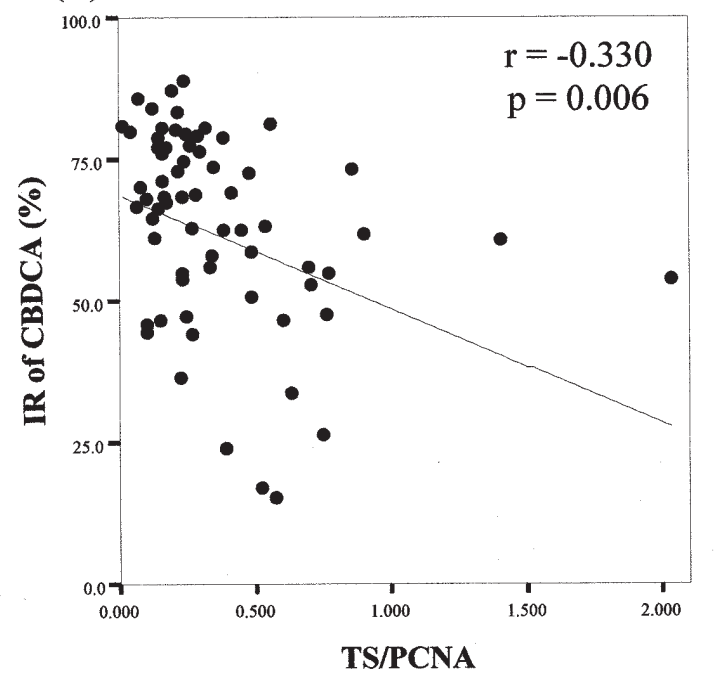

Figure 1. The level of TS/PCNA, the ratio of TS/actin and PCNA/actin, was correlated with the IRs of CDDP (A) and CBDCA (B) with correlation coefficients of $-0.274(\mathrm{p}=0.024)$ and $-0.330(\mathrm{p}=0.006)$, respectively.

This value represents the TS expression independent of cell cycle populations of analyzed cancer cells through normalization by G1 phase-specific gene expression of PCNA. DPD/ PCNA, the ratio of DPD/actin and PCNA/actin, was also calculated and the PCNA-normalized values were compared with IRs. The results indicated that the IRs of CDDP and CBDCA were linked to TS/PCNA and DPD/PCNA. The level of TS/PCNA was significantly correlated with the IRs of CDDP and CBDCA, with correlation coefficients of -0.274 $(p=0.024)$ and $-0.330(p=0.006)$, respectively (Fig. 1$)$. The level of DPD/PCNA was also significantly correlated with the IRs of CDDP and CBDCA, with correlation coefficients of -0.334 $(\mathrm{p}=0.005)$ and $-0.281(\mathrm{p}=0.021)$, respectively (Fig. 2). There were no relationships between the IRs of other anticancer drugs and PCNA-normalized TS or DPD mRNA expression.

Although TS polymorphism was not associated with IRs, it may affect the correlation between mRNA expression and IRs. Therefore, subgroup analyses were performed according

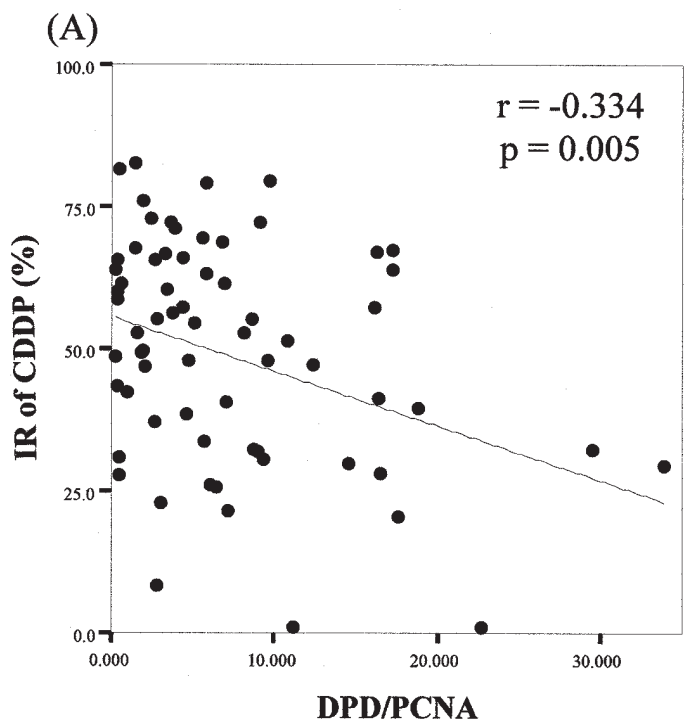

(B)

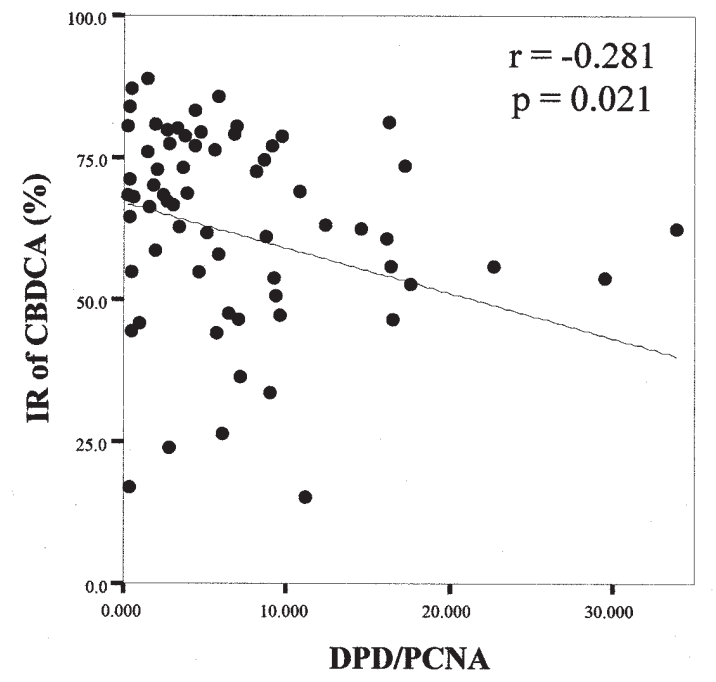

Figure 2. The level of DPD/PCNA, the ratio of DPD/actin and PCNA/actin, was correlated with the IRs of CDDP (A) and CBDCA (B) with correlation coefficients of $-0.334(\mathrm{p}=0.005)$ and $-0.281(\mathrm{p}=0.021)$, respectively.

to TS genotypes. The genotype was first classified by the TS VNTR. The results of subgroup analysis indicated that TS/PCNA was correlated with the IRs of CDDP, CBDCA, and $\mathrm{SN}-38$ ( $\mathrm{p}=0.019,0.010$, and 0.034 , respectively) in the $3 R / 3 R$ genotype group. DPD/PCNA was also correlated with the IR of CDDP ( $p=0.025$ ) when considering $3 R / 3 R$ genotype (Table III). There were no correlations of TS, DPD, or PCNA mRNA expression with the in vitro chemosensitivity in the $2 R$-containing genotype group $(2 R / 2 R$ and $2 R / 3 R)$. We then stratified the TS genotypes by the combination of VNTR and SNP, in which $3 \mathrm{G}$ was considered a high expression allele and $2 \mathrm{R}$ or $3 \mathrm{C}$ as low expression alleles according to the results of in vitro functional analysis reported previously (14). Based on this consideration, TS genotypes of $2 R / 3 G$, $3 \mathrm{C} / 3 \mathrm{G}$, and $3 \mathrm{G} / 3 \mathrm{G}$ were classified as high expression $(\mathrm{H})$ type and $2 \mathrm{R} / 2 \mathrm{R}, 2 \mathrm{R} / 3 \mathrm{C}$, or $3 \mathrm{C} / 3 \mathrm{C}$ as low expression (L) type. In the H-type group, TS/PCNA was correlated with the IRs of CDDP and CBDCA ( $\mathrm{p}=0.037$ and 0.006 , respectively), 
Table III. Association of the level of TS/PCNA and DPD/ PCNA with the IRs of CDDP, CBDCA, and SN-38 when considering the genotype of TS VNTR.

\begin{tabular}{lccc}
\hline & $\begin{array}{c}\text { Total } \\
(\mathrm{n}=68)\end{array}$ & $\begin{array}{c}2 \mathrm{R} / 2 \mathrm{R} \text { and } 2 \mathrm{R} / 3 \mathrm{R} \\
(\mathrm{n}=27)\end{array}$ & $\begin{array}{c}3 \mathrm{R} / 3 \mathrm{R} \\
(\mathrm{n}=40)\end{array}$ \\
\hline TS/PCNA & & & \\
CDDP & $\mathrm{r}=-0.274$ & $\mathrm{r}=-0.155$ & $\mathrm{r}=-0.370$ \\
& $\mathrm{p}=0.024$ & $\mathrm{p}=0.440$ & $\mathrm{p}=0.019$ \\
CBDCA & $\mathrm{r}=-0.330$ & $\mathrm{r}=-0.189$ & $\mathrm{r}=-0.404$ \\
& $\mathrm{p}=0.006$ & $\mathrm{p}=0.345$ & $\mathrm{p}=0.010$ \\
SN-38 & $\mathrm{r}=-0.168$ & $\mathrm{r}=0.069$ & $\mathrm{r}=-0.336$ \\
& $\mathrm{p}=0.170$ & $\mathrm{p}=0.732$ & $\mathrm{p}=0.034$ \\
DPD/PCNA & & & \\
CDDP & $\mathrm{r}=-0.334$ & $\mathrm{r}=-0.311$ & $\mathrm{r}=-0.354$ \\
& $\mathrm{p}=0.005$ & $\mathrm{p}=0.114$ & $\mathrm{p}=0.025$ \\
CBDCA & $\mathrm{r}=-0.281$ & $\mathrm{r}=-0.305$ & $\mathrm{r}=-0.264$ \\
& $\mathrm{p}=0.021$ & $\mathrm{p}=0.122$ & $\mathrm{p}=0.099$ \\
SN-38 & $\mathrm{r}=0.000$ & $\mathrm{r}=0.052$ & $\mathrm{r}=-0.014$ \\
& $\mathrm{p}=0.999$ & $\mathrm{p}=0.798$ & $\mathrm{p}=0.932$ \\
& & &
\end{tabular}

Table IV. Association of the level of TS/PCNA and DPD/ PCNA with the IRs of CDDP, CBDCA, and SN-38 when considering the genotype of G/C SNP.

\begin{tabular}{lccc}
\hline & $\begin{array}{c}\text { Total } \\
(\mathrm{n}=68)\end{array}$ & $\begin{array}{c}\text { H-type group } \\
(\mathrm{n}=42)\end{array}$ & $\begin{array}{c}\text { L-type group } \\
(\mathrm{n}=25)\end{array}$ \\
\hline $\begin{array}{l}\text { TS/PCNA } \\
\text { CDDP }\end{array}$ & $\mathrm{r}=-0.274$ & $\mathrm{r}=-0.323$ & $\mathrm{r}=-0.183$ \\
& $\mathrm{p}=0.024$ & $\mathrm{p}=0.037$ & $\mathrm{p}=0.380$ \\
CBDCA & $\mathrm{r}=-0.330$ & $\mathrm{r}=-0.420$ & $\mathrm{r}=-0.193$ \\
& $\mathrm{p}=0.006$ & $\mathrm{p}=0.006$ & $\mathrm{p}=0.355$ \\
SN-38 & $\mathrm{r}=-0.168$ & $\mathrm{r}=-0.245$ & $\mathrm{r}=-0.037$ \\
& $\mathrm{p}=0.170$ & $\mathrm{p}=0.117$ & $\mathrm{p}=0.862$ \\
DPD/PCNA & & & \\
CDDP & $\mathrm{r}=-0.334$ & $\mathrm{r}=-0.316$ & $\mathrm{r}=-0.321$ \\
& $\mathrm{p}=0.005$ & $\mathrm{p}=0.041$ & $\mathrm{p}=0.118$ \\
CBDCA & $\mathrm{r}=-0.281$ & $\mathrm{r}=-0.269$ & $\mathrm{r}=-0.251$ \\
& $\mathrm{p}=0.021$ & $\mathrm{p}=0.085$ & $\mathrm{p}=0.226$ \\
SN-38 & $\mathrm{r}=0.000$ & $\mathrm{r}=0.029$ & $\mathrm{r}=0.011$ \\
& $\mathrm{p}=0.999$ & $\mathrm{p}=0.857$ & $\mathrm{p}=0.959$ \\
\hline & & &
\end{tabular}

and DPD/PCNA with the IR of CDDP ( $\mathrm{p}=0.041)$ (Table IV). There were no correlations between TS, DPD, or PCNA mRNA expression with in vitro chemosensitivity in the L-type group.

\section{Discussion}

In the present study, we investigated the associations between in vitro sensitivity to anticancer drugs and TS polymorphism and TS, DPD, and PCNA mRNA expression in NSCLC to explore pharmacogenomic markers. The results indicated that there were no correlations between the IRs of any anticancer drugs and the TS polymorphism or TS, DPD, or PCNA mRNA expression. However, we observed associations between the IRs of platinum-derived drugs with the TS/PCNA, ratio of TS/actin and PCNA/actin, and with the DPD/PCNA, ratio of DPD/actin and PCNA/actin. Both TS/PCNA and DPD/PCNA were significantly correlated with the IRs of CDDP and CBDCA. It is uncertain why PCNA-normalized TS and DPD mRNA expression were associated with the efficacy of platinum-derived drugs. One possibility is that TS/PCNA and DPD/PCNA may represent the activity of nucleotide supply independent of cell cycle phase. TS and DPD are known to be expressed in a cell cycle-dependent manner, with higher levels of expression in S-phase (28). Therefore, TS and DPD mRNA expression increase with the size of the cell population in S-phase, which may conceal the roles of TS and DPD as indicators of nucleotide supply for DNA repair. On the other hand, PCNA-normalized mRNA expression levels can be used as cell cycle-independent markers because PCNA mRNA is expressed specifically at S-phase of the cell cycle. Taken together, these observations suggest that TS/PCNA and DPD/PCNA may be novel markers of the DNA repair capacity and thus may be linked with chemosensitivity to CDDP and CBDCA in lung cancer. This hypothesis should be tested further in large-scale clinical studies.

A number of clinical studies have indicated that TS and DPD expression can be used as novel predictive factors of the efficacy of 5-FU-based chemotherapy $(7,8)$. Therefore, we expected that TS or DPD mRNA expression would be useful as a marker of chemosensitivity to 5-FU in lung cancer. However, the results of the present study revealed no associations between chemosensitivity to 5-FU and TS or DPD mRNA expression. This result is not surprising because lung cancer is biologically distinct from gastrointestinal tumors, which were the subjects of previous studies indicting the pharmacogenomic significance of TS and DPD. However, 5-FU is generally ineffective in lung cancer (29), but effective in gastrointestinal cancer. There may be unknown resistance markers of 5-FU in lung cancer that are more important than TS and DPD for predicting chemosensitivity to 5-FU. Although we found no significant roles of TS or DPD expression as pharmacogenomic markers of 5-FU sensitivity, our results do not exclude further exploration of these markers in lung cancer. Current results of chemosensitivity testing are based on an in vitro culture system and MTT assay. It is well known that not all in vitro chemosensitivity data reflect the clinical response and that in vitro culture conditions of tumor cells can markedly influence the data. Higashiyama et al reported the relationships between TS and DPD activities in NSCLC with in vitro sensitivity to 5-FU (30). The inconsistency in their results with those of the present study may be attributed to differences in culture conditions and/or assay methods used to obtain chemosensitivity data. Fluoropyrimidine has been validated to be effective in lung cancer in an adjuvant setting (15-17). TS and DPD expression, as well as other pharmacogenomic factors, should be investigated further in the area of fluoropyrimidine therapy for lung cancer.

Although there was no correlation between TS polymorphism and sensitivity to anticancer drugs, correlations 
between the levels of TS/PCNA or DPD/PCNA and sensitivity to platinum-derived drugs were present in specific subgroups of TS polymorphism-i.e., the 3R/3R or H-type group. Tumors in either group were suggested to have higher translational activity of TS mRNA than the corresponding 2R-containing or L-type group. The correlation between TS expression and chemosensitivity may be dependent on the TS gene expression activity. Our previous study using gastric cancer cell lines showed similar effects of TS polymorphism. In this previous study, 3R/3R and 2R-containing groups showed different correlation curves between TS protein expression and sensitivity to 5-fluoro-5'-deoxyuridine (31). These results suggest that TS polymorphism and TS mRNA expression may be independent predictive factors of chemotherapy and should be analyzed in combination to improve the effectiveness of prediction.

With regard to the association between clinicopathological features and TS, DPD, and PCNA mRNA expression, the results indicated that adenocarcinoma tissues had higher levels of DPD/actin and lower levels of PCNA/actin than squamous cell carcinoma tissues. There were no relationships between TS, DPD, and PCNA mRNA expression and other clinicopathological features. Huang et al reported that squamous cell carcinoma showed higher levels of TS expression than adenocarcinoma detected immunohistochemically, while there was no significant difference in DPD expression (32). Higashiyama et al reported results similar to those described by Huang et al, although their results did not reach statistical significance (30). These reports and our results suggest that TS, DPD, and PCNA mRNA expression may have different ranges between adenocarcinoma and squamous cell carcinoma in lung cancer. This difference can affect the results of studies exploring pharmacogenomic markers in lung cancer. Therefore, samples homogeneous in histological type may need to be examined in future pharmacogenomic studies in lung cancer.

In conclusion, we observed the association between in vitro sensitivity to platinum-derived drugs with PCNA-normalized TS and DPD mRNA expression in NSCLC. These associations were affected by the TS polymorphism. These results warrant further large-scale clinical studies to clarify the role of TS genotyping and quantification of TS, DPD, and PCNA mRNA expression for prediction of the efficacy of chemotherapy in NSCLC.

\section{References}

1. Hoffman PC, Mauer AM and Vokes EE: Lung cancer. Lancet 355: 479-485, 2000.

2. Shirakusa $\mathrm{T}$ and Kobayashi K: Lung cancer in Japan: analysis of lung cancer registry for resected cases in 1994. Jpn J Lung Cancer 42: 555-566, 2002.

3. Mountain CF: Revisions in the international system for staging lung cancer. Chest 111: 1710-1717, 1997.

4. Non-small Cell Lung Cancer Collaborative Group: Chemotherapy in non-small cell lung cancer: a meta-analysis using updated data on individual patients from 52 randomised clinical trials. Br Med J 311: 899-909, 1995.

5. Scagliotti GV, Fossati R, Torri V, et al: Randomized study of adjuvant chemotherapy for completely resected stage I, II, or IIIA non-small cell Lung cancer. J Natl Cancer Inst 95: 1453-1461, 2003.

6. Danenberg PV: Thymidylate synthetase: a target enzyme in cancer chemotherapy. Biochim Biophys Acta 473: 73-92, 1977.

7. Yeh KH, Shun CT, Chen CL, et al: High expression of thymidylate synthase is associated with the drug resistance of gastric carcinoma to high dose 5-fluorouracil-based systemic chemotherapy. Cancer 82: 1626-1631, 1998.
8. Salonga D, Danenberg KD, Johnson M, et al: Colorectal tumors responding to 5-fluorouracil have low gene expression levels of dihydropyrimidine dehydrogenase, thymidylate synthase, and thymidine phosphorylase. Clin Cancer Res 6: 1322-1327, 2000.

9. Horie N, Aiba H, Oguro K, Hojo H and Takeishi K: Functional analysis and DNA polymorphism of the tandemly repeated sequences in the 5'-terminal regulatory region of the human gene for thymidylate synthase. Cell Struct Funct 20: 191-197, 1995.

10. Kawakami K, Omura K, Kanehira E and Watanabe Y: Polymorphic tandem repeats in the thymidylate synthase gene is associated with its protein expression in human gastrointestinal cancers. Anticancer Res 19: 3249-3252, 1999.

11. Kawakami K, Salonga D, Park JM, et al: Different lengths of a polymorphic repeat sequence in the thymidylate synthase gene affect translational efficiency but not its gene expression. Clin Cancer Res 7: 4096-4101, 2001.

12. Villafranca E, Okruzhnov Y, Dominguez MA, et al: Polymorphisms of the repeated sequences in the enhancer region of the thymidylate synthase gene promoter may predict downstaging after preoperative chemoradiation in rectal cancer. $\mathbf{J}$ Clin Oncol 19: 1779-1786, 2001

13. Iacopetta B, Grieu F, Joseph D and Elsaleh H: A polymorphism in the enhancer region of the thymidylate synthase promoter influences the survival of colorectal cancer patients treated with 5-fluorouracil. Br J Cancer 85: 827-830, 2001.

14. Kawakami $\mathrm{K}$ and Watanabe G: Identification and functional analysis of single nucleotide polymorphism in the tandem repeat sequence of thymidylate synthase gene. Cancer Res 63: 6004-6007, 2003.

15. Tanaka F, Miyahara R, Ohtake Y, Yanagihara K, Fukuse T, Hitomi S and Wada $\mathrm{H}$ : Advantage of post-operative oral administration of UFT (tegafur and uracil) for completely resected p-stage I-IIIa non-small cell lung cancer (NSCLC). Eur J Cardiothorac Surg 14: 256-262, 1998.

16. Endo C, Saito Y, Iwanami H, et al: North-east Japan Study Group for Lung Cancer Surgery: a randomized trial of postoperative UFT therapy in p stage I, II non-small cell lung cancer. Lung Cancer 40: 181-186, 2003.

17. Kato H, Ichinose Y, Ohta M, et al: Japan Lung Cancer Research Group on Postsurgical Adjuvant Chemotherapy: a randomized trial of adjuvant chemotherapy with uracil-tegafur for adenocarcinoma of the lung. N Engl J Med 350: 1713-1721, 2004.

18. Teixeira CR, Tanaka S, Haruma K, Yoshihara M, Sumii K and Kajiyama G: Proliferating cell nuclear antigen expression at the invasive tumor margin predicts malignant potential of colorectal carcinomas. Cancer 73: 575-579, 1994.

19. Tanaka S, Haruma K, Tatsuta S, et al: Proliferating cell nuclear antigen expression correlates with the metastatic potential of submucosal invasive colorectal carcinoma. Oncology 52: 134-139, 1995.

20. Zwelling LA and Kohn KW: Mechanism of action of cis-dichlorodiammineplatinum(II). Cancer Treat Rep 63: 1439-1444, 1979.

21. Shellard SA, Fichtinger-Schepman AM, Lazo JS and Hill BT: Evidence of differential cisplatin-DNA adduct formation, removal and tolerance of DNA damage in three human lung carcinoma cell lines. Anticancer Drugs 4: 491-500, 1993.

22. Scheithauer W, Clark GM, Salmon SE, Dorda W, Shoemaker RH and von Hoff DD: Model for estimation of clinically achievable plasma concentrations for investigational anticancer drugs in man. Cancer Treat Rep 70: 1379-1382, 1986.

23. Mosmann T: Rapid colorimetric assay for cellular growth and survival: application to proliferation and cytotoxicity assays. J Immunol Methods 65: 55-63, 1983.

24. Furukawa T, Kubota T, Watanabe M, et al: High in vitro-in vivo correlation of drug response using sponge-gel-supported threedimensional histoculture and the MTT end point. Int J Cancer 51: 489-498, 1992.

25. Furukawa T, Kubota T and Hoffman RM: Clinical applications of the histoculture drug response assay. Clin Cancer Res 1: 305-311, 1995.

26. Chomczynski P and Sacchi N: Single-step method of RNA isolation by acid guanidinium thiocyanate-phenol-chloroform extraction. Anal Biochem 162: 156-159, 1987.

27. Eads CA, Danenberg KD, Kawakami K, Saltz LB, Danenberg PV and Laird PW: CpG island hypermethylation in human colorectal tumors is not associated with DNA methyltransferase overexpression. Cancer Res 59: 2302-2306, 1999. 
28. Mirjolet JF, Barberi-Heyob M, Merlin JL, Marchal S, Etienne MC, Milano G and Bey P: Thymidylate synthase expression and activity: relation to S-phase parameters and 5-fluorouracil sensitivity. Br J Cancer 78: 62-68, 1998.

29. Weir AB III, Niell HB and Griffin JP: 5-Fluorouracil infusion and mitomycin combination chemotherapy in the management of patients with advanced non-small cell lung cancer. Am J Clin Oncol 12: 521-523, 1989.

30. Higashiyama M, Kodama K, Yokouchi H, et al: Thymidylate synthase and dihydropyrimidine dehydrogenase activities in non-small cell lung cancer tissues: relationship with in vitro sensitivity to 5-fluorouracil. Lung Cancer 34: 407-416, 2001.
31. Kaneko M, Kawakami K, Oyama K, Endo Y, Tanaka M, Sasaki T and Watanabe G: The association of expression and gene polymorphism of the thymidylate synthase gene with 5-fluoro5'-deoxyuridine sensitivity in gastric cancer cell lines. Jpn J Cancer Chemother 31: 1347-1350, 2004.

32. Huang CL, Yokomise H, Kobayashi S, Fukushima M, Hitomi S and Wada $\mathrm{H}$ : Intratumoral expression of thymidylate synthase and dihydropyrimidine dehydrogenase in non-small cell lung cancer patients treated with 5-FU-based chemotherapy. Int $\mathbf{J}$ Oncol 17: 47-54, 2000. 